\title{
Fatores que influenciam para transferência do cuidado intra-hospitalar efetiva:
}

\section{Revisão integrativa}

\author{
Factors that influence for effective intra-hospital care transfer: Integrative review \\ Factores que influyen para una transferencia efectiva de atención intrahospitalaria: Revisión
}

integrativa

Recebido: 12/07/2021 | Revisado: 19/07/2021 | Aceito: 21/07/2021 | Publicado: 28/07/2021

Thailini Silva de Mello

ORCID: https://orcid.org/0000-0003-0023-6163 Universidade Federal de Santa Maria, Brasil

E-mail: thailinimello@gmail.com

Jeanini Dalcol Miorin

ORCID: https://orcid.org/0000-0001-7672-7191

Universidade Federal de Santa Maria, Brasil

E-mail: jeaninidmiorin@hotmail.com

Silvamar Camponogara

ORCID: https://orcid.org/0000-0001-9342-3683

Universidade Federal de Santa Maria, Brasil

E-mail: silviaufsm@yahoo.com.br

Cristiane Cardoso de Paula

ORCID: https://orcid.org/0000-0003-4122-5161

Universidade Federal de Santa Maria, Brasil

E-mail: cris_depaula1@ @otmail.com

Camila Pinno

ORCID: https://orcid.org/0000-0002-9145-1987 Hospital da Brigada Militar de Santa Maria, Brasil

E-mail: pinnocamila@gmail.com

Etiane de Oliveira Freitas

ORCID: https://orcid.org/0000-0002-8589-2524

Universidade Federal de Santa Maria, Brasil

E-mail: etiof@yahoo.com.br

\begin{abstract}
Resumo
Objetivo: identificar na literatura os fatores que influenciam para realização da transferência do cuidado intrahospitalar efetiva pela equipe de enfermagem. Metodologia: trata-se de uma revisão integrativa de literatura, realizada em três bases de dados: Web of Science, Medical Literature Analysis and Retrieval System Online (MEDLINE) e Sci Verse Scopus (SCOPUS). Foram incluídos estudos primários, em português, espanhol ou inglês, publicados entre os anos de 2015 a 2021. Resultados: a análise criteriosa dos 39 estudos internacionais possibilitou identificar que a realização de transferências relacionadas a pacientes adultos e pediátricos e realizadas por meio do deslocamento ou não do paciente no ambiente intra-hospitalar sofrem influência de fatores como a comunicação, transporte, trabalho em equipe e educação em saúde e estes repercutem na efetividade da transferência do cuidado intra-hospitalar. Conclusão: diante da análise dos estudos encontrados nota-se a comunicação como um fator predominante, embora os fatores transporte, trabalho em equipe e educação em saúde também apresentam características significativas que influenciam na realização da transferência do cuidado intra-hospitalar efetiva.

Palavras-chave: Transferência de pacientes; Transferência da responsabilidade pelo paciente; Enfermagem; Assistência hospitalar.

Abstract

Objective: to identify in the literature the factors that influence the transfer of effective intra-hospital care by the nursing team. Methodology: this is an integrative literature review, carried out in three databases: Web of Science, Medical Literature Analysis and Retrieval System Online (MEDLINE) and Sci Verse Scopus (SCOPUS). Primary studies were included, in Portuguese, Spanish or English, published between the years 2015 to 2021. Results: the careful analysis of 39 international studies made it possible to identify that transfers related to adult and pediatric patients and carried out through the displacement or not of the patient in the intra-hospital environment are influenced by factors such as communication, transport, teamwork and health education, and these impact on the effectiveness of the transfer of intra-hospital care. Conclusion: in view of the analysis of the studies found, communication is seen as a predominant factor, although the transport, teamwork and health education factors also present significant
\end{abstract}


characteristics that influence the realization of effective intra-hospital care transfer.

Keywords: Patient transfer; Patient handoff; Nursing; Hospital care.

\begin{abstract}
Resumen
Objetivo: identificar en la literatura los factores que influyen en la transferencia de una atención intrahospitalaria efectiva por parte del equipo de enfermería. Metodología: se trata de una revisión integradora de la literatura, realizada en tres bases de datos: Web of Science, Medical Literature Analysis and Retrieval System Online (MEDLINE) y Sci Verse Scopus (SCOPUS). Se incluyeron estudios primarios, en portugués, español o inglés, publicados entre 2015 y 2021. Resultados: el análisis detenido de 39 estudios internacionales permitió identificar que los traslados relacionados con pacientes adultos y pediátricos y realizados a través del desplazamiento o no del paciente en el ámbito intrahospitalario están influenciados por factores como la comunicación, el transporte, el trabajo en equipo y educación en salud, y estos afectan la efectividad de la transferencia de la atención intrahospitalaria. Conclusión: a la vista del análisis de los estudios encontrados, la comunicación se ve como un factor predominante, aunque los factores de transporte, trabajo en equipo y educación para la salud también presentan características significativas que influyen en la realización de una transferencia asistencial intrahospitalaria efectiva.
\end{abstract}

Palabras clave: Transferencia de pacientes; Pase de Guardia; Enfermería; Atención hospitalaria.

\title{
1. Introdução
}

O ambiente hospitalar é caracterizado como uma complexa organização dos serviços de saúde (Pinheiro et al., 2017). Envolve diversos profissionais, que trabalham em um mesmo local, com diferentes rotinas e frequentemente com infraestrutura inadequada e falta de recursos humanos e materiais (Pinheiro et al., 2017; Alves \& Melo, 2019). Por esses motivos, diversos são os fatores e situações que predispõem a ocorrência de ações que resultam em dano ao paciente, ou seja, eventos adversos no ambiente hospitalar (Brasil, 2014).

Nessa perspectiva, a segurança do paciente, é o movimento que preza pela realização de serviços assistenciais com qualidade e segurança, uma vez que, as possíveis falhas trazem impactos negativos para saúde do paciente, bem como para o processo de trabalho (Brasil, 2017). Frente a isso, entende-se que as preocupações em relação a práticas seguras envolvem diversos aspectos assistenciais, sendo, a transferência do cuidado uma das atividades potencialmente vulneráveis para a segurança do paciente.

A transferência do cuidado é parte essencial na dinâmica do atendimento. Ela está relacionada com o fluxo seguro no trabalho hospitalar e é uma importante etapa para garantir o processo sequencial, sucessivo e ininterrupto que acompanha o paciente nos serviços de saúde, ou seja, na continuidade do cuidado, a fim de garantir a não fragmentação, a qualidade da assistência e a segurança do paciente desde sua admissão até a alta (Raeisi et al., 2019).

É definida pela transferência das responsabilidades do cuidado de um paciente ou de um grupo de pacientes, para um profissional ou para um grupo de profissionais de forma definitiva ou temporária (Alves et al., 2019). Portanto, a transferência de cuidado envolve a responsabilidade que os profissionais têm pela qualidade da assistência prestada e das ações que garantam a sucessão do cuidado por meio de uma prática efetiva (Fealy et al., 2019).

Nesse contexto, a transferência do cuidado intra-hospitalar efetiva, é descrita como uma atividade assistencial crítica, que envolve fatores, ou seja, elementos que influenciam na qualidade da execução da troca das responsabilidades pelos cuidados dos pacientes entre os profissionais de saúde. Esses fatores, portanto, quando executados corretamente, garantem a realização de uma transferência do cuidado efetiva e que resulta na continuidade do cuidado (Menser et al., 2010). Diante disso, pesquisas comprovam que ao implementarem um checklist eletrônico, simples e estruturado, ocorreu uma melhora em 75\% na qualidade das transferências intra-hospitalares (Agarwala et al., 2015). Como também, a utilização de um processo sistematizado de transferência do cuidado demonstrou resultados de melhora na satisfação dos enfermeiros em relação ao processo de trabalho e a comunicação envolvendo a transferência do cuidado (Talley et al., 2019).

Entretanto, diversos são os desafios para realizar essa atividade de maneira efetiva. Muitas transferências do cuidado são realizadas por relações desconexas entre equipes de entrega e recebimento do cuidado de pacientes, tempo de transferência 
entre unidades prolongado, trocas de informações incompletas e realizadas em ambientes em que os profissionais de enfermagem são facilmente distraídos ou interrompidos por outros profissionais ou por toques de telefone e falta de ferramentas e protocolos institucionais para efetivar a execução dessa atividade (Raeisi et al., 2019; Fealy et al., 2019). Desse modo, transferências do cuidado ineficazes geram a probabilidade de $80 \%$ de falhas, comprometendo a continuidade da assistência, a qualidade do cuidado e segurança do paciente (Alves \& Melo, 2019; Raeisi et al., 2019; Agarwala et al., 2015).

Diante dessa problemática, é possível afirmar que transferências do cuidado intra-hospitalares quando realizadas de forma efetiva tendem a viabilizar a continuidade do cuidado e, consequentemente, maior segurança para o paciente e melhor qualidade da assistência. Ainda, acredita-se que evidenciar os fatores que contribuem para transferências do cuidado efetivas podem colaborar para avaliação desta prática e posterior construção de protocolos que visem a qualificação dessa atividade. Portanto, o objetivo desse estudo foi identificar na literatura os fatores que influenciam para realização da transferência do cuidado intra-hospitalar efetiva pela equipe de enfermagem.

\section{Metodologia}

Trata-se de uma revisão integrativa de literatura, a qual consiste na busca, avaliação crítica e a síntese das evidências disponíveis sobre o determinado tema a ser investigado. Para a condução desse tipo de revisão busca-se determinar o objetivo específico por meio da elaboração de questionamentos pertinentes; encontrar na literatura pesquisas consideradas relevantes e que se enquadram nos critérios de inclusão previamente elaborados; análise criteriosa do método utilizado nos estudos selecionados; exploração dos dados coletados sintetizando-os e conclusão para findar a execução da revisão integrativa (Mendes et al., 2008).

Para elaboração da pergunta de revisão foi utilizado o mnemônico PCC sugerido pelo JBI Manual for Evidence Synthesis (Aromataris \& Munn, 2020), significando P: população, C: conceito e C: contexto. Correspondendo a: P: equipe de enfermagem, C: transferência do cuidado efetiva e C: intra-hospitalar. A questão de pesquisa norteadora é: Quais os fatores que influenciam para realização da transferência do cuidado intra-hospitalar efetiva pela equipe de enfermagem?

A busca dos estudos foi realizada em novembro de 2020 e fevereiro de 2021, nas bases de dados Web of Science, Medical Literature Analysis and Retrieval System Online (MEDLINE) e Sci Verse Scopus (SCOPUS), instituindo o recorte temporal de 2015 a 2021. Para a escolha dos Medical Subject Headings (MeSH) e Descritores em Ciências da Saúde (DECs), de modo a ampliar a recuperação de documentos nas bases de dados, foi desenvolvido o mapeamento dos descritores/palavraschave de artigos que versavam sobre a temática. A estratégia de busca elaborada nas bases de dados Web of Science e SCOPUS foi composta pelos seguintes descritores: patient transfer, patient handoff, handoff, nursing e hospital care seguidos pelo operador booleano "AND" e na base MEDLINE foram usados os descritores: transferência de pacientes, transferência de responsabilidade pelo paciente, transferência, enfermagem e assistência hospitalar seguidos de "AND".

A seleção dos artigos foi baseada nos critérios de inclusão, que consistiam em ser estudos no idioma inglês, espanhol ou português, estar publicado dentro do período de tempo definido e versar sobre o tema respondendo à questão de pesquisa. Foram excluídos estudos de revisões, teses e dissertações. Artigos repetidos foram contados apenas uma vez.

A avaliação dos estudos selecionados de acordo com os critérios de inclusão propostos foi executada por duas pesquisadoras. Quando ocorreram discordâncias em relação aos estudos selecionados, foi realizada a análise em conjunto, buscando-se um consenso. Para a operacionalização das buscas, foi seguido o modelo proposto pelo JBI (Aromataris et al., 2020), representado na Figura 1.

Foram encontrados 261 estudos primários, sendo 49 publicações na SCOPUS, 51 na Web of Science e 161 estudos na MEDILINE. Aplicado os critérios de seleção e a delimitação temporal de 2015 a 2020, foram selecionados 51 artigos para realizar a leitura na íntegra. Após a leitura íntegra e criteriosa dos artigos foi elegido um total de 39 estudos primários das três 
bases, SCOPUS, Web of Science e MEDLINE, sendo respectivamente quatro, 17 e 18. A exclusão de 12 publicações, após a leitura minuciosa, se justifica por serem produções que não respondem à questão de pesquisa.

Figura 1 - PRISMA do processo da seleção dos estudos

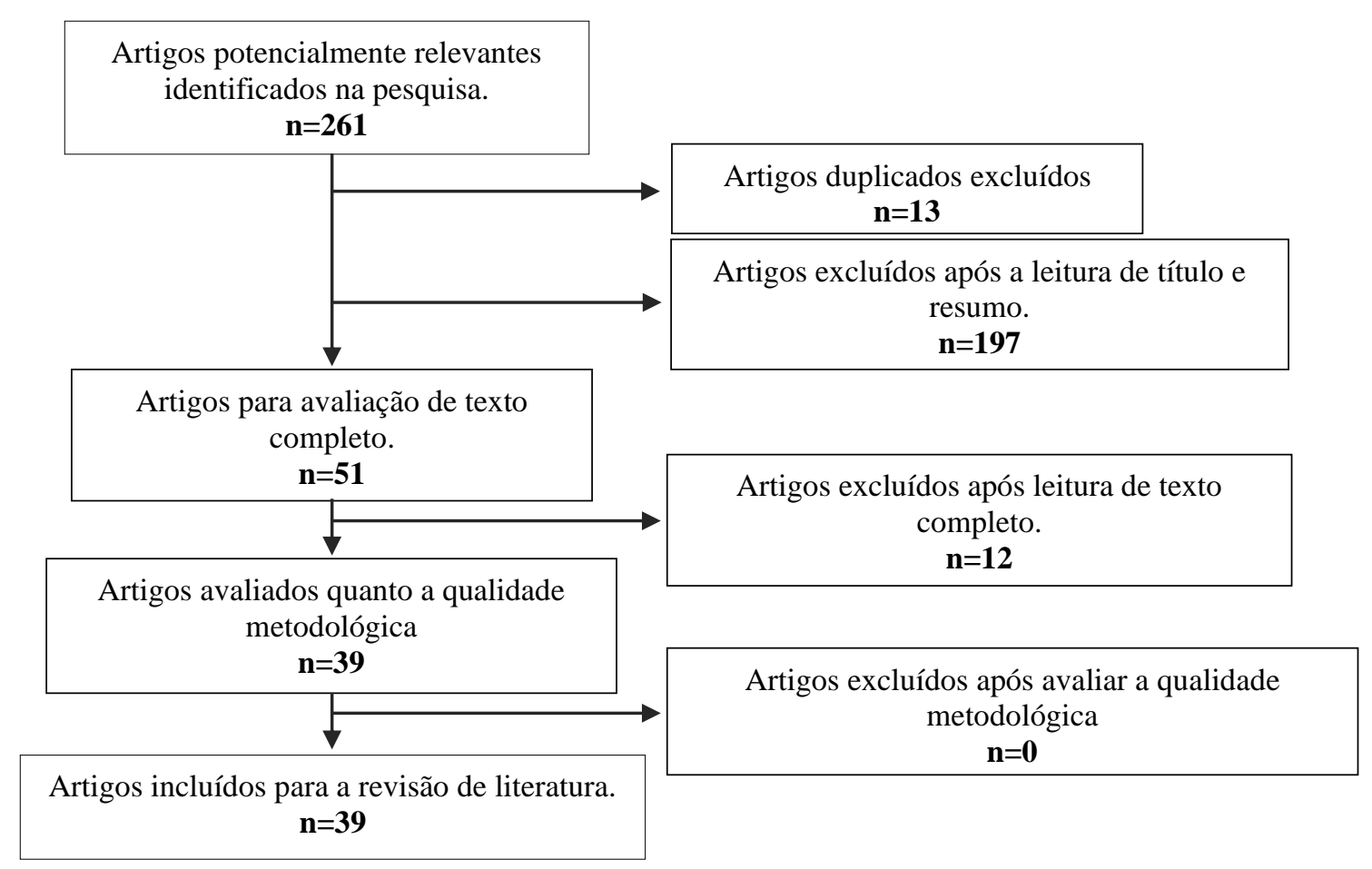

Fonte: Adaptado do JBI Manual for Evidence Synthesis (2020)

Os níveis de evidências dos estudos foram caracterizados por meio de uma perspectiva hierárquica. Dessa forma, para determinar o nível de evidência a aplicação da hierarquia considerou o tipo de questão de pesquisa do estudo primário: Intervenção ou tratamento, Prognóstico ou etiologia e Significado e a força de evidência foi classificada conforme a abordagem metodológica do estudo (Melnyk et al., 2011).

A compilação dos dados extraídos das produções incluídas para este estudo, foi colocada em um documento compondo os seguintes itens: identificação das bases de dados, ano de publicação, autores, título do artigo, periódico, local da pesquisa, questão de pesquisa, delineamento metodológico, nível de evidência, principais resultados e conclusão. Após, foram dissertadas as informações de modo que permitissem convergir com o objetivo proposto no estudo.

\section{Resultados}

A caracterização das produções incluídas $(n=39)$ mostrou que a maioria $(28,2 \%)$ dos estudos foram publicados no ano de 2017, seguidas do ano de 2019 (17,95\%) e 2018 (17,95\%), $2020(12,8 \%)$ e $2015(12,8 \%)$ e o ano de 2016 com o menor quantitativo (10,26\%). Em relação a questão de pesquisa, a maior parte das produções $(38,46 \%)$ apresentam a questão voltada para significado (Melnyk et al., 2011). Em relação aos países onde os estudos foram realizados, todos foram internacionais, sendo o Estados Unidos o país com predomínio (38,46\%) das publicações, seguido da Austrália (12,26\%), Canadá (10,26\%), China e Suécia (7,69\%) cada, Grécia (5,13\%) e Japão, França, Espanha, Escócia, Suíça, Irã e Singapura (2,56\%) cada. A seguir a apresentação da caracterização dos estudos no Quadro 1. 
Quadro 1 - Caracterização das produções incluídas no estudo. Rio Grande do Sul, Brasil, 2021.

\begin{tabular}{|c|c|c|}
\hline Id. & Resultados/Conclusão & $\begin{array}{l}\text { Delineamento/Tip } \\
\text { o de Estudo }\end{array}$ \\
\hline $\begin{array}{l}\text { Popovici et } \\
\text { al., } 2015\end{array}$ & $\begin{array}{l}\text { Problemas são identificados no processo de transferência de pacientes e as } \\
\text { ferramentas de comunicação usadas durante esse processo implicam na qualidade } \\
\text { da comunicação, como também, podem resultar em dificuldades imprevistas com } \\
\text { o uso de novas tecnologias. }\end{array}$ & $\begin{array}{l}\text { Qualitativo; } \\
\text { Significado-N2 }\end{array}$ \\
\hline $\begin{array}{l}\text { Zakrison et } \\
\text { al., } 2016\end{array}$ & $\begin{array}{l}\text { Discrepâncias de informações ocorreram em } 48 \% \text { das transferências devido ao } \\
\text { fornecimento de informações divergentes, falta de liderança e a falta de uma } \\
\text { estrutura formalizada para troca de informações. Dessa forma, conhecer os } \\
\text { membros da equipe e padronizar as transferências é necessário para melhorar a } \\
\text { qualidade e a segurança do paciente. }\end{array}$ & $\begin{array}{l}\text { Misto; Siginificado- } \\
\text { N4 }\end{array}$ \\
\hline $\begin{array}{l}\text { Clairol et al., } \\
2017\end{array}$ & $\begin{array}{l}\text { Foram identificadas deficiências de conteúdo, como: não declaração de alergias, } \\
\text { escala de Glasgow, avaliação da dor, contenção mecânica, entre outros. A } \\
\text { padronização de repasse poderia minimizar as deficiências e melhorar a segurança } \\
\text { do paciente. }\end{array}$ & $\begin{array}{l}\text { Coorte; Prognóstico } \\
\text { ou etiologia-N2 }\end{array}$ \\
\hline $\begin{array}{l}\text { Gu et al., } \\
2018\end{array}$ & $\begin{array}{l}\text { Sete fatores foram elencados e as características se relacionam resumidamente a } \\
\text { segurança do paciente e comunicação. Diferentes percepções foram apontadas } \\
\text { sobre o processo de transferências entre as unidades como: treinamento de } \\
\text { transferências, educação e comunicação. }\end{array}$ & $\begin{array}{l}\text { Quantitativo; } \\
\text { Significado-N2 }\end{array}$ \\
\hline $\begin{array}{l}\text { Agarwala et } \\
\text { al., } 2015\end{array}$ & $\begin{array}{l}\text { O desenvolvimento de uma lista de verificação, resultou em melhorias, } \\
\text { principalmente na frequência de retransmissão de informações e qualidade da } \\
\text { comunicação na transferência. }\end{array}$ & $\begin{array}{l}\text { Coorte; Intervenção } \\
\text { ou tratamento-N } 4\end{array}$ \\
\hline $\begin{array}{l}\text { Fabila et al., } \\
2016\end{array}$ & $\begin{array}{l}\text { O mnemônico SBAR foi reformatado para esclarecer ambiguidades, transmitir } \\
\text { informações ideias e promover facilidade no uso. As mudanças geraram melhores } \\
\text { resultados na comunicação, aprimorando a retransmissão de conteúdo. }\end{array}$ & $\begin{array}{l}\text { Coorte; Intervenção } \\
\text { ou tratamento-N4 }\end{array}$ \\
\hline $\begin{array}{l}\text { Segall et al., } \\
2016\end{array}$ & $\begin{array}{l}\text { Antes e após a implementação do novo modelo as mudanças significativas } \\
\text { ocorreram em relação ao comportamento da equipe de trabalho e satisfação da } \\
\text { equipe, reduzindo a carga de trabalho sem aumentar o tempo das transferências de } \\
\text { pacientes. }\end{array}$ & $\begin{array}{l}\text { Coorte; Intervenção } \\
\text { ou tratamento-N4 }\end{array}$ \\
\hline $\begin{array}{l}\text { Yang et al., } \\
2016\end{array}$ & $\begin{array}{l}\text { Após a implementação de um protocolo de transferência a comunicação e trabalho } \\
\text { em equipe melhoraram repercutindo também em melhorias relacionadas ao } \\
\text { cuidado. }\end{array}$ & $\begin{array}{l}\text { Coorte; Intervenção } \\
\text { ou tratamento-N4 }\end{array}$ \\
\hline $\begin{array}{l}\text { Bergman et } \\
\text { al., } 2017\end{array}$ & $\begin{array}{l}\text { O transporte intra-hospitalar é um processo perigoso para pacientes criticamente } \\
\text { enfermos, vários fatores tanto humanos quanto materias podem contribuir para } \\
\text { eventos adversos relacionados ao transporte. }\end{array}$ & $\begin{array}{l}\text { Coorte; Prospectivo } \\
\text { ou etiologia-N2 }\end{array}$ \\
\hline $\begin{array}{l}\text { Jullia et al., } \\
2017\end{array}$ & $\begin{array}{l}\text { Antes do treinamento e com a implementação de uma lista, as pontuações nos } \\
\text { grupos intervenção e controle foram semelhantes. Com a intervenção a pontuação } \\
\text { aumentou e a melhora persistiu por meses e sem aumento da duração das } \\
\text { transferências. O treinamento e o uso da lista melhoram os cuidados na realização } \\
\text { de transferências. }\end{array}$ & $\begin{array}{l}\text { Coorte; Intervenção } \\
\text { ou tratamento-N4 }\end{array}$ \\
\hline $\begin{array}{l}\text { Nugus et al., } \\
2017\end{array}$ & $\begin{array}{l}\text { O estudo identifica cinco competências de comunicação de complexidade } \\
\text { crescente e fatores para a comunicação como: hierarquia, limites organizacionais } \\
\text { formalmente impostos e papéis, poder e educação que contribuem para garantir a } \\
\text { transferência do paciente, esses fatores são expressos e moldam a comunicação. }\end{array}$ & $\begin{array}{l}\text { Qualitativo; } \\
\text { Significado-N2 }\end{array}$ \\
\hline $\begin{array}{l}\text { Sanchezet al., } \\
2017\end{array}$ & $\begin{array}{l}\text { A implementação de um modelo de transferência procurou maximizar a } \\
\text { oportunidade de desconexão assíncrona e, ao mesmo tempo, proporcionar a } \\
\text { oportunidade de desconexão verbal quando julgada necessária. }\end{array}$ & $\begin{array}{l}\text { Coorte Intervenção } \\
\text { ou tratamento-N4 }\end{array}$ \\
\hline $\begin{array}{l}\text { Thomson et } \\
\text { al., } 2018\end{array}$ & $\begin{array}{l}\text { Ao compreender os fatores que contribuem para a transferência, foi possível } \\
\text { desenvolver intervenções que visem melhorar a qualidade da comunicação na } \\
\text { transferência. }\end{array}$ & $\begin{array}{l}\text { Quantitativo; } \\
\text { Significado-N4 }\end{array}$ \\
\hline $\begin{array}{l}\text { Yegane et al., } \\
2017\end{array}$ & $\begin{array}{l}\text { Após o treinamento do uso da ISBAR nos processos de transferência houve uma } \\
\text { melhora em todos os parâmetros da ferramenta em comparação a fase do estudo } \\
\text { antes da intervenção. }\end{array}$ & $\begin{array}{l}\text { Coorte; Intervenção } \\
\text { ou tratamento-N4 }\end{array}$ \\
\hline $\begin{array}{l}\text { Yu et al., } \\
2017\end{array}$ & $\begin{array}{l}\text { A pesquisa mostrou uma melhora nos critérios propostos, além disso, demonstrou } \\
\text { que transferências podem ser mais eficazes ao traduzir as evidências práticas por } \\
\text { meio de auditoria baseada em evidências. Ressalta, também, o requerimento de } \\
\text { ferramentas padronizadas na transferência entre turnos. }\end{array}$ & $\begin{array}{l}\text { Coorte; Intervenção } \\
\text { ou tratamento-N4 }\end{array}$ \\
\hline Lautz et al., & $\mathrm{O}$ treinamento estruturado de transferência e o fornecimento de uma ajuda & Coorte; Intervenção \\
\hline
\end{tabular}




\begin{tabular}{|c|c|c|}
\hline 2018 & $\begin{array}{l}\text { cognitiva podem melhorar a inclusão de informações essenciais do paciente na } \\
\text { transferência de crianças simuladas em estado crítico. }\end{array}$ & ou tratamento-N4 \\
\hline $\begin{array}{l}\text { Padgett et al., } \\
2018\end{array}$ & $\begin{array}{l}\text { O uso do SBAR afetou positivamente as percepções de comunicação dos } \\
\text { enfermeiros durante as transferências de pacientes. }\end{array}$ & \begin{tabular}{|l|} 
Coorte; \\
Intervenção \\
tratamento-N4
\end{tabular} \\
\hline $\begin{array}{l}\text { Ramsay et } \\
\text { al., } 2018\end{array}$ & $\begin{array}{l}\text { A introdução da folha de transferência tornou o processo mais suave e melhorou a } \\
\text { segurança do paciente e a qualidade do atendimento. }\end{array}$ & $\begin{array}{l}\text { Coorte; Intervenção } \\
\text { ou tratamento-N4 }\end{array}$ \\
\hline $\begin{array}{l}\text { VanGraafeila } \\
\text { nd et al., } \\
2018\end{array}$ & $\begin{array}{l}\text { Padronização por meio da gravidade da doença e ferramentas de comunicação } \\
\text { reduzem os riscos à segurança. Houve percepção de que menos dados foram } \\
\text { omitidos ou perdidos durante o novo processo de entrega e transporte. }\end{array}$ & \begin{tabular}{|l|} 
Qualitativo; \\
Intervenção \\
tratamento-N6
\end{tabular} \\
\hline $\begin{array}{l}\text { France et al., } \\
2019\end{array}$ & $\begin{array}{l}\text { Os eventos não rotineiros ocorrem em uma alta taxa e são de gravidade variável no } \\
\text { cuidado perioperatório neonatal. Os profissionais entrevistados recomendam } \\
\text { transferências estruturadas para todos os pacientes. }\end{array}$ & $\begin{array}{l}\text { Coorte; Prognóstico } \\
\text { ou etiologia-N2 }\end{array}$ \\
\hline $\begin{array}{l}\text { Rikos et al., } \\
2019\end{array}$ & $\begin{array}{l}\text { Os participantes dizem que a transferência de enfermagem, deve incluir, } \\
\text { principalmente, instruções médicas, condição geral de cada paciente, } \\
\text { administração de medicamentos e resultados de enfermagem. Muito poucas } \\
\text { enfermeiras acreditavam que deveriam incluir a condição hemodinâmica do } \\
\text { paciente, condição psicológica, ambiente familiar e planejamento da assistência de } \\
\text { enfermagem. }\end{array}$ & $\begin{array}{l}\text { Qualitativo; } \\
\text { Significado-N2 }\end{array}$ \\
\hline $\begin{array}{l}\text { O' Connor et } \\
\text { al., } 2020\end{array}$ & $\begin{array}{l}\text { Lacunas na comunicação do enfermeiro foram identificadas como fatores que } \\
\text { impactam negativamente a comunicação de transferência do enfermeiro, exigindo } \\
\text { soluções alternativas. }\end{array}$ & $\begin{array}{l}\text { Qualitativo; } \\
\text { Significado-N2 }\end{array}$ \\
\hline $\begin{array}{l}\text { Caruso et al., } \\
2015\end{array}$ & $\begin{array}{l}\text { A abordagem padronizada e baseada em equipe para transferências de sala de } \\
\text { cirurgia para SRPA aumentou a quantidade de informações do paciente transferido } \\
\text { e a satisfação do enfermeiro da SRPA e não aumentou a duração da transferência. }\end{array}$ & $\begin{array}{l}\text { Coorte; Intervenção } \\
\text { ou tratamento-N4 }\end{array}$ \\
\hline $\begin{array}{l}\text { McMullan et } \\
\text { al., } 2015\end{array}$ & $\begin{array}{l}\text { A comunicação foi altamente interativa ao discutir o estado do paciente e planos } \\
\text { de cuidados futuros. Os enfermeiros fizeram perguntas de forma proativa para } \\
\text { capturar uma grande proporção das informações de que precisavam. }\end{array}$ & $\begin{array}{l}\text { Qualitativo; } \\
\text { Significado-N2 }\end{array}$ \\
\hline $\begin{array}{l}\text { Moon et al., } \\
2016\end{array}$ & $\begin{array}{l}\text { A implementação de uma intervenção de transferência esteve associada ao } \\
\text { aumento da satisfação da equipe de enfermagem, maior eficiência e qualidade no } \\
\text { processo geral de transferência. }\end{array}$ & $\begin{array}{l}\text { Coorte; Intervenção } \\
\text { ou tratamento-N4 }\end{array}$ \\
\hline $\begin{array}{l}\text { Bakon et al., } \\
2017\end{array}$ & $\begin{array}{l}\text { A implementação de um formulário de transferência foi positiva. Os participantes } \\
\text { indicaram que o formulário é claro, bem desenhado e fácil de navegar. Ele } \\
\text { forneceu instruções para padronizar a transferência clínica e aumentar a } \\
\text { responsabilidade e a responsabilização neste processo. }\end{array}$ & $\begin{array}{l}\text { Mistos; Intervenção } \\
\text { ou tratamento-N6 }\end{array}$ \\
\hline $\begin{array}{l}\text { Randmmaa et } \\
\text { al., } 2017\end{array}$ & $\begin{array}{l}\text { As diferentes percepções dos profissionais sobre a transferência pós-operatória } \\
\text { indicaram que as intervenções são necessárias para minimizar a lacuna entre as } \\
\text { percepções e práticas para alcançar um entendimento compartilhado da } \\
\text { transferência pós-operatória. }\end{array}$ & $\begin{array}{l}\text { Qualitativo; } \\
\text { Significado-N2 }\end{array}$ \\
\hline $\begin{array}{l}\text { Stelfox et al., } \\
2017\end{array}$ & $\begin{array}{l}\text { As transferências são caracterizadas por falhas no fluxo e de comunicação. As } \\
\text { recomendações de melhoria incluíram um plano de cuidados documentado para } \\
\text { ser encaminhado com o paciente, transferência face a face padronizada, e } \\
\text { informar os pacientes sobre transferências pendentes com antecedência. }\end{array}$ & $\begin{array}{l}\text { Coorte; Prognóstico } \\
\text { ou etiológico-N2 }\end{array}$ \\
\hline $\begin{array}{l}\text { Gu et al., } \\
2018\end{array}$ & $\begin{array}{l}\text { Cinco fatores de transferência foram derivados. Os participantes mostraram uma } \\
\text { boa compreensão de seus próprios papéis e comunicação adequada durante as } \\
\text { transferências. A qualidade geral da transferência foi afetada principalmente pela } \\
\text { comunicação mútua, pelo sistema de transferência e pelo ambiente. }\end{array}$ & $\begin{array}{l}\text { Quantitativo; } \\
\text { Significado-N4 }\end{array}$ \\
\hline $\begin{array}{l}\text { Rikos et al., } \\
2018\end{array}$ & $\begin{array}{l}\text { Foram identificadas } 30 \text { características formando quatro grupos: o uso da prática } \\
\text { baseada em evidências, o não uso da prática baseada em evidências e sua } \\
\text { correlação com ambiente psicológico tenso, gerenciamento de pacientes e as } \\
\text { habilidades / conhecimentos clínicos de enfermeiros e transferência qualidade da } \\
\text { informação transferida. }\end{array}$ & $\begin{array}{l}\text { Qualitativo; } \\
\text { Significado-N2 }\end{array}$ \\
\hline $\begin{array}{l}\text { Rosonberg et } \\
\text { al., } 2018\end{array}$ & $\begin{array}{l}\text { Foi possível identificar diferentes microssistemas culturais no ambiente hospitalar, } \\
\text { nos quais as transferências se moldam e possuem identidades de acordo com a sua } \\
\text { unidade. }\end{array}$ & $\begin{array}{l}\text { Qualitativo; } \\
\text { Significado-N2 }\end{array}$ \\
\hline $\begin{array}{l}\text { Germack et } \\
\text { al., } 2020\end{array}$ & $\begin{array}{l}\text { Entre as condições de cooperação e conflito, foram identificados que, as políticas } \\
\text { de fluxo de pacientes criam desequilíbrios de poder, relacionamentos foram úteis } \\
\text { para facilitar transferências seguras e discordância no método do pedido de } \\
\text { admissão. }\end{array}$ & $\begin{array}{l}\text { Qualitativo; } \\
\text { Significado-N2 }\end{array}$ \\
\hline
\end{tabular}




\begin{tabular}{|c|c|c|}
\hline $\begin{array}{l}\text { Hada et al., } \\
2019\end{array}$ & $\begin{array}{l}\text { Barreiras: incerteza sobre o compartilhamento de informações, lacunas de } \\
\text { comunicação, pouco envolvimento do paciente, limitações de tempo e desafios } \\
\text { ambientais. E como facilitadores, o uso de prontuários eletrônicos integrados, } \\
\text { respaldo e expectativas claras da liderança de enfermagem e educação voltada para } \\
\text { a transferência. }\end{array}$ & $\begin{array}{l}\text { Qualitativo; } \\
\text { Significado-N2 }\end{array}$ \\
\hline $\begin{array}{l}\text { Hendrickson } \\
\text { et al., } 2019\end{array}$ & $\begin{array}{l}\text { A teleconferência no compartilhamento de informações na transferência de } \\
\text { cuidados é uma alternativa bem aceita. Os participantes acreditam que ele apoia o } \\
\text { atendimento seguro ao paciente. }\end{array}$ & $\begin{array}{l}\text { Quantitativo; } \\
\text { Intervenção } \\
\text { tratamento-N4 }\end{array}$ \\
\hline $\begin{array}{l}\text { Talley et al., } \\
2019\end{array}$ & $\begin{array}{l}\text { Após a implementação de um processo de transferência multidisciplinar } \\
\text { sistemático, os enfermeiros relataram uma melhora na frequência e integridade do } \\
\text { processo de transferência, resultando em uma percepção de melhor atendimento ao } \\
\text { paciente. }\end{array}$ & $\begin{array}{l}\text { Coorte; Intervenção } \\
\text { ou tratamento-N4 }\end{array}$ \\
\hline $\begin{array}{l}\text { Fekieta et al., } \\
2020\end{array}$ & $\begin{array}{l}\text { Fatores considerados importantes para transferências bem-sucedidas: } \\
\text { desenvolvimento e aplicação de protocolos; robustez da tecnologia; trabalho em } \\
\text { equipe; capacidade hospitalar. São fatores que ampliam a compreensão das } \\
\text { transferências e informam o desenvolvimento de um guia prático. }\end{array}$ & $\begin{array}{l}\text { Qualitativo; } \\
\text { Significado-N2 }\end{array}$ \\
\hline $\begin{array}{l}\text { Tacchini- } \\
\text { Jacquier et } \\
\text { al., } 2020\end{array}$ & $\begin{array}{l}\text { Os resultados do estudo caracterizaram os requisitos percebidos para um padrão de } \\
\text { transferência em enfermagem. }\end{array}$ & $\begin{array}{l}\text { Qualitativo; } \\
\text { Significado-N4 }\end{array}$ \\
\hline $\begin{array}{l}\text { Tobiano et } \\
\text { al., } 2020\end{array}$ & $\begin{array}{l}\text { A transferência dentro do hospital requer o compartilhamento de informações de } \\
\text { qualidade. A continuidade relacional entre os enfermeiros é uma consideração } \\
\text { importante ao melhorar a transferência intra-hospitalar. }\end{array}$ & $\begin{array}{l}\text { Qualitativo; } \\
\text { Significado-N2 }\end{array}$ \\
\hline $\begin{array}{l}\text { Vretare et al., } \\
2020\end{array}$ & $\begin{array}{l}\text { O complexo fenômeno que é a transferência de enfermagem reflete na sua } \\
\text { compreensão de várias formas ao se referir a comunicação entre equipe, } \\
\text { oportunidade de aprendizagem, coleta de informações, responsabilidade e } \\
\text { segurança do paciente. }\end{array}$ & $\begin{array}{l}\text { Qualitativo; } \\
\text { Significado-N2 }\end{array}$ \\
\hline
\end{tabular}

Fonte: Autores (2021).

Foi possível identificar que 43,59\% (n=17) dos estudos são relacionados as transferências em que os pacientes não são deslocados, ou seja, transferências do cuidado que ocorrem entre os profissionais da mesma unidade de assistência, por exemplo, durante a passagem de plantão. Já nas transferências em que ocorre o deslocamento do paciente entre diferentes unidades de cuidado, os estudos apontaram que a maioria $15,38 \%(\mathrm{n}=6)$ das transferências acontecem do departamento de emergência para as unidades de internação. Além disso os estudos encontrados envolvem o processo de transferências do cuidado realizado por profissionais que trabalham na assistência de pacientes adultos e pediátricos, sendo 84,61\% ( $\mathrm{n}=33$ ) estudos relacionados ao cuidado a pacientes adultos e $15,38 \%(n=6)$ direcionados aos os cuidados a pacientes pediátricos.

A transferência é uma atividade que envolve a coordenação e o fluxo do paciente durante os processos assistenciais dentro de um mesmo serviço de saúde ou entre diferentes níveis de cuidado e utiliza de diversos fatores que podem interferir na sua execução. Por essa razão, autores destacam alguns fatores para realização de transferências do cuidado efetivas, com vistas a possibilitar a continuidade do cuidado e a troca das responsabilidades pelo paciente (Alves \& Melo, 2019; Merten et al., 2017). Ao analisar os estudos foi possível identificar quatro fatores: comunicação ( $n=23)$, transporte ( $n=1)$, trabalho em equipe ( $n=3)$ e educação em saúde $(n=1)$, conforme representado na Figura 2. Ainda, alguns estudos apresentaram dois fatores para se referirem a realização da transferência do cuidado, como: comunicação e trabalho em equipe $(\mathrm{n}=7)$, comunicação e educação em saúde ( $n=3)$ e transporte e trabalho em equipe $(n=1)$. 
Figura 2 - Fatores que influenciam na realização de transferências do cuidado intra-hospitalar efetivas, Rio Grande do Sul, Brasil, 2021.

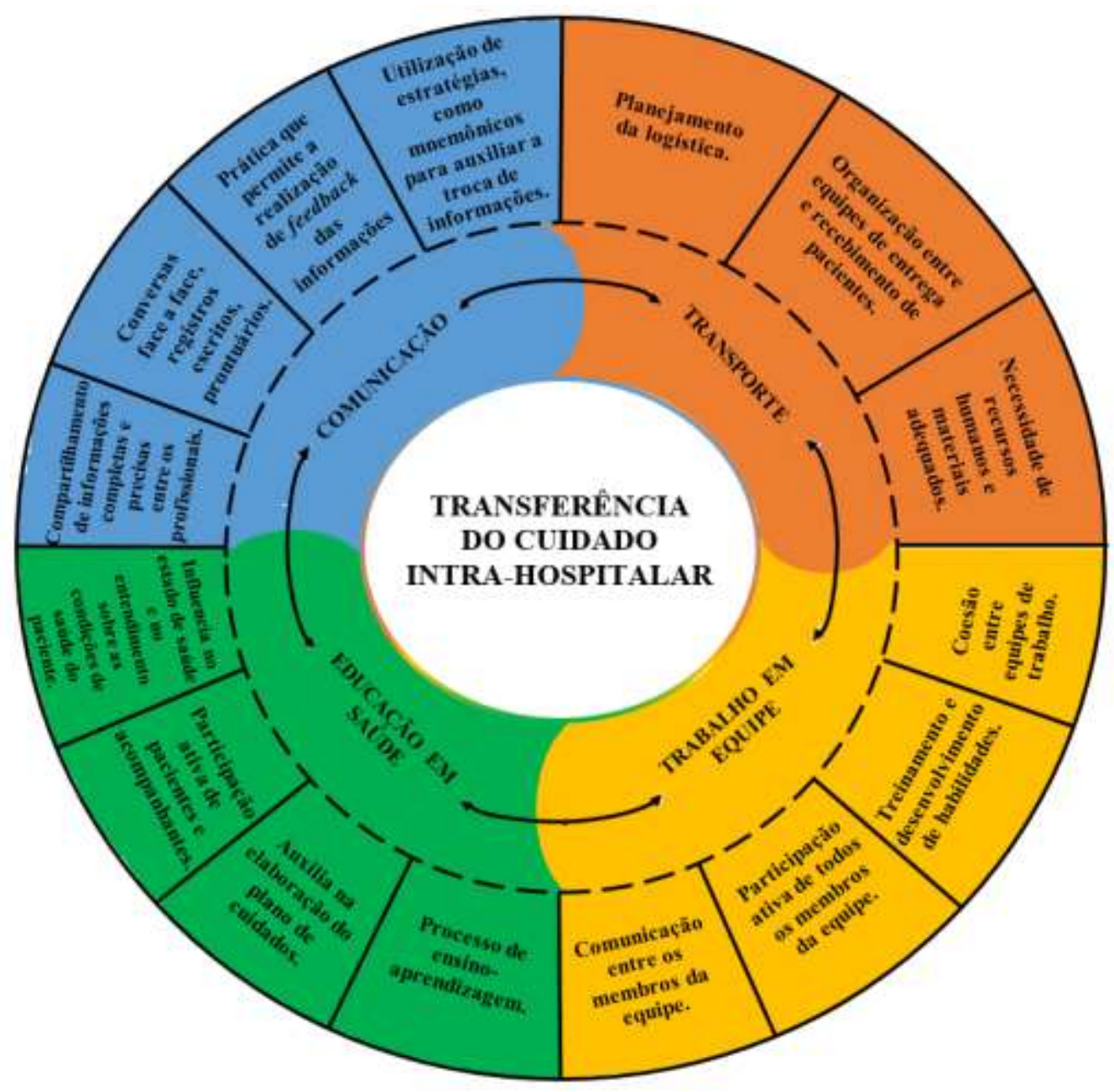

Fonte: Autores (2021).

Ressalta-se que a comunicação foi um fator predominante nos estudos encontrados, entretanto os demais fatores também apresentam relevância ao pensar que transferências do cuidado intra-hospitalares se relacionam com as condições de trabalho, interrupções e distrações, coesão entre equipes, equipamentos utilizados para o transporte do paciente e o envolvimento de pacientes e acompanhantes no cuidado (Randmaa et al., 2017).

\section{Discussão}

As trasferências de cuidado são práticas rotineiras nos serviços de saúde, visto que, um paciente pode ser transferido até 15 vezes durante uma hospitalização de cinco dias e um profissional de saúde pode participar de 3000 transferências em um mês, sendo que, muitas vezes, elas são realizadas sem o estabelecimento de padronizações ou uso de protocolos (TacchiniJacquier et al., 2020; Merten et al., 2017). Por esse motivo, a transferência do cuidado é uma atividade que exige treinamento dos profissionais, colaboração interprofissional, organização, tomada de decisão e precisão e eficiência na entrega e recebimento dos cuidados dos pacientes (Menser et al., 2008; Germack et al., 2020). 
Nesse sentido, Fealy et al. (2019) evidenciam que transferências efetivas são viabilizadas por meio da padronização, treinamento de equipes e uso de protocolos institucionais que possam contribuir para a realização cada vez mais adequada dos fatores que influenciam na transferência do cuidado (Menser et al., 2010). Dessa maneira, os artigos incluídos no presente estudo vincularam a realização da transferência do cuidado intra-hospitalar efetiva com os fatores: comunicação, transporte, trabalho em equipe e educação em saúde.

A comunicação, considerada um dos principais elementos da transferência do cuidado, é utilizada para compreender e atender as necessidades dos pacientes, coordenar o cuidado e partilhar informações (Tacchini-Jacquier et al., 2020). Além disso, a troca de informações entre os profissionais de saúde, durante a transferência do cuidado, é uma etapa fundamental, pois é por meio da qualidade da comunicação que as informações relevantes são compartilhadas e compreendidas pelos profissionais (Humphries et al., 2019). Frente a isso, autores definem a comunicação como a passagem de informações de um indivíduo para o outro através de qualquer canal de comunicação, como: conversa face a face, telefone, registros escritos, fontes de informações formal e prontuários (Popovici et al., 2015).

Estudo afirma que a transferência do cuidado se utiliza, em grande parte, da comunicação, sendo que as diferentes formas de trocas de informações têm associação com o aumento da probabilidade de eventos adversos, visto que poucos enfermeiros verificam se o receptor soube interpretar as informações corretamente (Vretare et al., 2020). Além disso, autores apontam a realização do feedback das informações com o objetivo de minimizar eventos adversos a que se relacionam. Corroborando, Argawala et al. (2015) disserta sobre a implementação de uma ferramenta eletrônica simples e estruturada com itens essenciais para a transferência do cuidado intraoperatório. A ferramenta tem como fim, promover a transmissão e a retenção de informações mais relevantes e específicas de modo a melhorar a comunicação entre a equipe, promover uma troca de informações mais qualificada e melhorar a satisfação dos profissionais na realização da transferência do cuidado.

A Joint Commission International e a Organização Mundial da Saúde, afirmam que uma das melhores maneiras para realizar uma comunicação segura e efetiva nas transferências do cuidado é com uso de ferramentas padronizadas, como o mnemônico SBAR, que significa: Situation /situação em que o paciente se encontra; Background /antecedentes clínicos; Assessment/ avaliação do profissional sobre o paciente e Recommendation/ recomendações do profissional para continuidade do cuidado. Ele é uma das ferramentas mais utilizadas para estruturar a troca de informações entre os profissionais, melhorando a comunicação e a segurança do paciente durante as transferências do cuidado (WHO et al., 2007; Patient Safety Movement, 2019).

Além da comunicação, o transporte intra-hospitalar também é parte fundamental para realização de transferências do cuidado efetivas, principalmente quando há o deslocamento do paciente entre os serviços hospitalares. Definido como o encaminhamento temporário ou definitivo de pacientes, por profissionais de saúde dentro do ambiente hospitalar, seja para fins de diagnósticos ou terapêuticos, o transporte de pacientes é considerado uma importante atividade da transferência do cuidado, por envolver organização, comunicação, logística, suporte material, recursos humanos, padronização do procedimento, capacitação profissional e homogeneização da equipe (Fanara et al., 2010).

Dessa maneira, o transporte de pacientes é um período associado a ocorrência de incidentes, uma vez que requer a ação sincronizada entre as equipes de envio e recebimento do paciente e realocação física do mesmo (VanGraafeiland et al., 2018). Estudo realizado sobre o transporte de pacientes da unidade de tratamento intensivo para unidades de tratamento e diagnóstico aponta que o transporte intra-hospitalar é um processo delicado, pois apresenta riscos para os pacientes, principalmente quando realizados com aqueles em condições instáveis de saúde. Complicações como: instabilidade hemodinâmica, aumento da pressão intracraniana, padrões respiratórios alterados, hiper ou hipoglicemia, entre outros, podem ocorrer com os mesmos no momento em que estão sendo transportados e agravar ainda mais seu estado de saúde (Bergman et al., 2017). 
O transporte intra-hospitalar está relacionado com o planejamento e atuação organizada das equipes de cuidado ao paciente, bem como da escolha de equipamentos adequados. Estudo feito na Suécia mostra que um terço (34\%) dos perigos relacionados ao transporte de pacientes se associam ao mau uso dos equipamentos, ferramentas e tecnologias (Bergman et al., 2017). Assim, o treinamento e aperfeiçoamento constante dos profissionais envolvidos, bem como a padronização das ações e os recursos materiais necessários para a monitoração clínica do paciente devem estar à disposição para a prevenção ou minimização de eventos adversos (Fanara et al., 2010).

Frente a essa necessidade de garantir a transferência do cuidado de maneira efetiva, outro fator que possibilita a coordenação e continuidade do cuidado é o trabalho em equipe (Ramsay et al., 2018; Segall et al., 2016; Yang et al., 2016;). Conceituado por ser uma atividade que conecta diferentes processos de trabalho, valorizando os membros da equipe na produção de cuidados e construindo consensos e objetivos a serem alcançados coletivamente, o trabalho em equipe reflete como é o engajamento das equipes na realização da transferência do cuidado (Navarro et al., 2013).

O trabalho em equipe é considerado uma parte complexa da transferência do cuidado, visto que, em muitas situações, envolve o relacionamento entre equipes de diferentes unidades de cuidado, o que designa um desafio para realização de transferências efetivas (Germack et al., 2020; Humphries et al., 2019). Frente a isso, O'Connor et al. (2020), afirmam que a dificuldade de trabalhar em equipe, durante as transferências do cuidado, está associada a falta de conhecimento sobre os cuidados a serem realizados por cada membro das equipes e por informações divergentes entre as mesmas, repercutindo em ações desorganizadas para o recebimento e a continuidade da assistência prestada ao paciente.

Diante da necessidade de realizar a transferência do cuidado intra-hospitalar efetiva, além da comunicação, transporte e trabalho em equipe, a educação em saúde é um fator indispensável para a qualidade dessa atividade. A educação em saúde é entendida como um dos principais elementos durante a transferência do cuidado, implicando na troca de conhecimento entre profissionais, pacientes e acompanhantes com a intenção de possibilitar o envolvimento desses como parceiros do cuidado. ${ }^{(4,56)}$ Estudo ratifica que a não inclusão do paciente e acompanhantes no processo de transferência do cuidado pode reduzir as percepções dos enfermeiros sobre a situação social de seus pacientes limitando a sua capacidade de determinar a presença de fatores que influenciam a saúde dos mesmos (Bakon \& Millichamp, 2017).

Autores apontam que inclusão de um elemento que garantisse, ao enfermeiro, discutir o plano de cuidados com o paciente seria essencial para a transferência do cuidado, pois coloca ênfase o envolvimento do paciente. Este envolvimento é fundamental para reduzir falhas de comunicação e eventos adversos. Os pacientes precisam se envolver nos planos de tratamento pretendidos e possuir a opção de esclarecer qualquer uma de suas preocupações, pois a realização da educação em saúde possibilita a troca de conhecimento sobre as condições e cuidados de saúde (Bakon \& Millichamp, 2017).

Em vista disso, ao realizar a transferência do cuidado intra-hospitalar o profissional deve inserir paciente e acompanhantes no plano de cuidados e certificar-se de que os mesmos estão compreendendo o processo (Merten et al., 2017). As responsabilidades, intervenções e elaboração de planos de cuidados devem ser compartilhadas entre profissionais de enfermagem, pacientes e acompanhantes, dado que, quando estes são incluídos na transferência do cuidado eles sentem-se mais seguros e mais receptivos às intervenções que estão ocorrendo ou aos planos de cuidados a serem aplicados, ficando mais confiantes na melhora do quadro clínico (Rikos et al., 2018).

Visto que nessa revisão integrativa de literatura os estudos incluídos abordam a educação em saúde apenas para com os pacientes e seus acompanhantes, vê se a necessidade da discussão de estudos que versem sobre a educação em saúde ou educação permanente para com os profissionais no contexto da transferência do cuidado intra-hospitalar. $\mathrm{O}$ educar, ensinar, faz parte do cotidiano das organizações do trabalho por meio $d$ a elaboração de técnicas, tecnologias e planejamento dessa atividade (Delatorre et al., 2013). A educação permanente se baseia numa política pedagógica que é realizada no contexto 
laboral, implicando na importância da qualificação dos trabalhadores, pois as ações desenvolvidas devem basear-se nas necessidades do seu público com o objetivo de possibilitar transformar e qualificar as práticas profissionais (Brasil, 2018).

Frente ao exposto, nota-se a transferência do cuidado como parte da rotina de enfermagem, importante para o compartilhamento da assistência, além de servir como base para o planejamento e execução do cuidado (Vretare et al., 2020). É uma atividade que demanda competências, habilidades e conhecimentos da equipe de enfermagem, sendo considerada uma prática de riscos para o fluxo do trabalho (Alsolamy et al., 2018). Portanto, é um processo assistencial de suma importância e que envolve a articulação de tais fatores para garantir a coordenação, a continuidade e segurança do cuidado.

Por fim, considera-se uma limitação deste estudo os descritores utilizados, dado que são poucos usados no Brasil e por esse fato estudos brasileiros não foram encontrados para essa revisão de literatura. Como contribuições, é um dos primeiros estudos brasileiros que aborda quatro fatores principais para a transferência do cuidado intra-hospitalar efetiva. Os resultados deste estudo fornecerão subsídios para avaliação das transferências do cuidado intra-hospitalares realizadas pelas equipes de enfermagem.

\section{Conclusão}

Diante da análise dos estudos sobre a temática da transferência do cuidado, vale ressaltar que a comunicação é o fator predominante relacionado a essa prática assistencial. Entretanto, fatores como transporte, trabalho em equipe e educação em saúde apresentam características importantes que contribuem para realização de transferências do cuidado intra-hospitalares efetivas.

Nessa revisão integrativa de literatura apesar de ter sido encontrado um número expressivo de estudos, todos foram produções internacionais, limitando assim uma análise da realidade nacional da presente produção. Ainda pode se considerar limitado, a escassez de estudos sobre demais fatores que venham a influenciar na transferência do cuidado intra-hospitalar efetiva, como também se considera pouco quantitativo de estudos que discutem sobre transporte, trabalho em equipe e educação e saúde na transferência do cuidado intra-hospitalar quando comparados com o fator comunicação.

Tendo em vista isso, revela-se a necessidade da produção de estudos nacionais acerca da temática e também ressaltamos a carência de estudos que versem sobre outros fatores relacionados a transferência do cuidado para além da comunicação. A produção deste estudo tem como fim ampliar a visão de profissionais de saúde sobre a realização da transferência do cuidado, qualificar o fluxo do trabalho, a continuidade do cuidado e instigar que demais estudos possam vir a contribuir com essa prática assistencial.

\section{Referências}

Agarwala, A. V., Firth, P. G., Albrecht, M. A., Warren, L. \& Musch, G. (2015). An electronic checklist improves transfer and retention of critical information at intraoperative handoff of care. Anesth Analg, 120(1),96-104. https://doi.org/10.1213/ANE.0000000000000506

Alsolamy, S., Al-Sabhan, A., Alassin, N., Sadat, M., Qasim, E. A., Tamim, H. \& Arabi, Y. M. (2018). Management and outcomes of patients presenting with sepsis and septic shock to the emergency department during nursing handover: a retrospective cohort study. BMC Emerg Med, 18(3). https://doi.org/10.1186/s12873-018-0155-8

Alves, M. \& Melo C. L. (2019) A transferência do cuidado na perspectiva de profissionais de enfermagem de um pronto-socorro. Revista Mineira de Enfermagem, 23(e), 1194. http://www.dx.doi.org/10.5935/1415-2762.20190042

Aromataris, E. \& Munn, Z (Editores). (2020). JBI Manual for Evidence Synthesis. JBI, 2020. https://doi.org/10.46658/JBIMES-20-01

Bakon, S. \& Millichamp, T. (2017). Optimising the emergency to ward handover process: A mixed methods study. Australas Emerg Nurs J, 20(4), 147-152. https://doi.org/10.1016/j.aenj.2017.10.001

Bergman, L. M., Pettersson, M. E., Chaboyer, W. P., Carlström, E. D. \& Ringdal, M. L. (2017). Safety Hazards During Intrahospital Transport: A Prospective Observational Study. Crit Care Med, 45(10), e1043-e1049. http://dx.doi.org/10.1097/CCM.0000000000002653 
Brasil. Agência Nacional de Vigilância Sanitária. (2017). Assistência Segura: Uma Reflexão Teórica Aplicada à Prática Agência Nacional de Vigilância Sanitária. Brasília: Anvisa, $2^{\mathrm{a}} \quad$ ed. 2017. http://portal.anvisa.gov.br/documents/33852/3507912/Caderno+1+-+Assistencia+Segura++Uma+Reflexao+Teorica+Aplicada+a+Pratica/97881798-cea0-4974-9d9b077528ea1573.

Brasil. Ministério da Saúde. (2014). Documento de referência para o Programa Nacional de Segurança do Paciente / Ministério da Saúde, Fundação Oswaldo Cruz, Agência Nacional de Vigilância $\quad$ Sanitária. $\quad-\quad$ Brasília: $\quad$ Ministério da Saúde. https://bvsms.saude.gov.br/bvs/publicacoes/documento_referencia_programa_nacional_seguranca.pdf.

Brasil. Ministério da Saúde. (2018). Secretaria de Gestão do Trabalho e da Educação na Saúde. Planejamento das Ações de Educação Permanente em Saúde no Sistema Único de Saúde: Orientações / Ministério da Saúde, Secretaria de Gestão do Trabalho e da Educação na Saúde, Departamento de Gestão da Educação na Saúde - Brasília: Ministério da Saúde. http://bvsms.saude.gov.br/bvs/publicacoes/orientacoes_planejamento_acoes_educacao_permanente.pdf.

Caruso, T. J., Marquez, J. L., Wu, D. S., Shaffer, J. Á., Balise, R. R., Groom, M., ... Sharek, P. J. (2015). Implementation of a standardized postanesthesia care handoff increases information transfer without increasing handoff duration. Jt Comm J Qual Patient Saf, 41(1), 35-42. https://doi.org/10.1016/S1553$7250(15) 41005-0$

Clairol, M. G., Ordozgoiti, A. V., Lugo, D. O., Maspoch, E. C., Font, M. M. \& Oliva, M. E. (2017). Evaluación deltraspaso de información (Hand Off) en equipos de enfermería de urgências. Revista Cubana de Enfermería, 33(3), 1-17. http://www.revenfermeria.sld.cu/index.php/enf/article/view/1539/292

Delatorre, P. G., Sá, S. P. C., Valente, G. S. C. \& Silvino, Z. R. (2013). Planejamento para alta hospitalar como estratégia de cuidado de enfermagem: revisão integrativa. Revista de Enfermagem UEPE, 7(esp), 7157-9. https://doi.org/10.5205/1981-8963-v7i12a12387p7151-7159-2013

Fabila, T. S., Hee, H. I., Sultana, R., Assam, P. N., Kiew, A. \& Chan, Y. H. (2016). Improving postoperative handover from anaesthetists to non-anaesthetists in a children's intensive care unit: the receiver's perception. Singapore Med J, 57(5), 242-53. http://dx.doi.org/10.11622/smedj.2016090

Fanara, B., Manzon, C., Barbot, O., Desmettre, T. \& Capellier, G. (2010). Recommendations for the intra-hospital transporto f critically ill patients. Critical Care Medicine, 14(3), R87. https://doi.org/10.1186/cc9018

Fealy, G., Donnelly, S., Doyle, G., Brenner, M., Hughes, M., Mylotte, E., \& Zaki, M. (2019). Clinical handover practices among healthcare practitioners in acute care services: A qualitative study. J Clin Nurs, 28(1-2), 80-88. https://doi.org/10.1111/jocn.14643

Fekieta, R., Rosenberg, A., Hodshon, B., Feder, S., Chaudhry, S. I. \& Emerson, B. L. (2020). Organisational factors underpinning intra-hospital transfers: a guide for evaluating contexto in quality improvement. Health Systems. https://doi.org/10.1080/20476965.2020.1768807.

France, D. J., Slagle, J., Schremp, E., Moroz, S., Hatch, L. D., Grubb, P., \& Blakely, M. L. (2019). Impact of patient handover structure on neonatal perioperative safety. J Perinatol, 39(3), 453-467. https://doi.org/10.1038/s41372-018-0305-6

Germack, H. D., Fekieta, R., Britton, M. C., Feder, S. L., Rosenberg, A. \& Chaudhry, S. I. (2020). Cooperation and conflict in intra-hospital transfer: A qualitative analysis. Nursing Open, 7, 634-641. https://doi.org/10.1002/nop2.434

Gu, X. \& Itoh, K. (2018). How Nurses Perceive Organizational Climate Surrounding Patient Handoffs in Japanese Hospitals? Springer. https://link.springer.com/chapter/10.1007/978-3-319-96089-0_59.

Gu, X., Liu, H. G. \& Itoh, K. (2018). Inter-department patient handoff quality and its contributing factors in Chinese hospitals. Cognition, Technology \& Work, 21, 133-143. https://doi.org/10.1007/s10111-018-0500-4

Hada, A., Jack, L. \& Coyer, F. (2019). Using a knowledge translation framework to identify barriers and supports to effective nursing handover: A focus group study. Heliyon, 5(6). https://doi.org/10.1016/j.heliyon.2019.e01960

Hendrickson, M. A., Schempf, E. M., Furnival, R. A., Marmet, J., Lunos, A. S. \& Jacob, A. K. (2019). The Admission Conference Call: A Novel Approach to Optimizing Pediatric Emergency Department to Admitting Floor Communication. Jt Comm J Qual Patient Saf, 45(6), 431-439. https://doi.org/10.1016/j.jcjq.2019.02.008

Humphries, C., Jaganathan, S., Panniyammakal, J., Singh, S. K., Goenka, S., Dorairaj, P., ... Manaseku-Holland, S. (2019). Patient and healthcare provider knowledge, attitudes and barriers to handover and healthcare communication during chronic disease inpatient care in India: a qualitative exploratory study. BMJ Open, 11,9(11), e028199. http://dx.doi.org/10.1136/bmjopen-2018-028199

Jullia, M., Tronet, A., Fraumar, F., Minville, V., Fourcade, O., Alacoque, X., \& Kurrek, M. M. (2017). Training in intraoperative handover and display of a checklist improve communication during transfer of care: An interventional cohort study of anaesthesia residents and nurse anaesthetists. Eur J Anaesthesiol, 34(7), 471-476. 10.1097/EJA.0000000000000636

Lautz, A. J., Martin, K. C., Nishisaki, A., Bonafide, C. P., Hales, R. L., Hunt, E. A., ... Boyer, D. L. (2018). Focused Training for the Handover of Critical Patient Information During Simulated Pediatric Emergencies. Hosp Pediatr, 8(4), 227-231. https://doi.org/10.1542/hpeds.2017-0173

McMullan, A., Parush, A. \& Momtahan, K. (2015). Transferring patient care: patterns of synchronous bidisciplinary communication between physicians and nurses during handoffs in a critical care unit. J Perianesth Nurs, 30(2), 92-104. https://doi.org/10.1016/j.jopan.2014.05.009

Melnyk, BM. \& Fineout-overholt E. (2011). Evidence-based practice in nursing \& healthcare. A guide to best practice. 2ed. Philadelphia: Wolters Kluwer, Lippincop Williams \& Wilkins,2011.

Mendes, K. S., Silveira, R. C. C. P. \& Galvão, C. M. (2008). Revisão integrativa: método de pesquisa paraa incorporação de evidências na saúde e na enfermagem. Texto contexto - enferm, 17(4), 758-764. https://doi.org/10.1590/S0104-07072008000400018.

Menser, T., Foster, S., Gisin, S., Jaeckel, D. \& Ummenhofer, W. (2010). Assessing the quality of patient handoffs at care transitions. Qual Saf Health Care, 19(6), e44. http://dx.doi.org/10.1136/qshc.2009.038430

Merten, H., Van Galen, L. S. \& Wagner, C. (2017). Safe Handover: practical guidance. British medical jornal, 359, j4328. https://doi.org/10.1136/bmj.j4328 
Moon, T. S., Gonzales, M. X., Woods, A. P. \& Fox, P. E. (2016). Improving the quality of the operating room to intensive care unit handover at an urban teaching hospital through a bundled intervention. J Clin Anesth, 31, 5-12. https://doi.org/10.1016/j.jclinane.2016.01.001

Navarro, A. S. S., Guimarães, R. L. S. \& Garanhani, M. L. (2013). Trabalho em equipe: o significado atribuído por profissionais da estratégia de saúde da família. Revista Mineira de Enfermagem, 17(1), 61-68. http://www.dx.doi.org/10.5935/1415-2762.20130006

Nugus, P., McCarthy, S., Holdgate, A., Braithwaite, J., Schoenmakers, A. \& Wagner, C. (2017). Packaging Patients and Handing Them Over: Communication Context and Persuasion in the Emergency Department. Ann Emerg Med, 69(2), 210-217.e2. https://doi.org/10.1016/j.annemergmed.2016.08.456

O’Connor, D. T., Rawson, H. \& Redley, B. (2020). Nurse-to-nurse communication about multidisciplinary care delivered in the emergency department: An observation study of nurse-to-nurse handover to transfer patient care to general medical wards. Australasian Emergency Care, 23(Issue 1), 37-46. https://doi.org/10.1016/j.auec.2019.12.004.

Padgett, T. M. (2018). Improving Nurses' Communication During Patient Transfer: A Pilot Study. J Contin Educ Nurs, 49(8), 378-384. https://doi.org/10.3928/00220124-20180718-09

Patient Safety Movement. (2019). Instituto Brasileiro para a Segurança do Paciente. Como usar o método SBAR na transição do cuidado. https://www.segurancadopaciente.com.br/qualidade-assist/como-usar-o-metodo-sbarna-transicao-do-cuidado/.

Pinheiro, M. P. \& Junior, O. C. S. (2017). Evaluación de la cultura de seguridad del paciente en una organización hospitalaria de un hospital universitario. Enfermería Global, 45, 335-338. https://doi.org/10.6018/eglobal.16.1.238811

Popovici, I., Morita, P. P., Doran, D., Lapinsky, S., Morra, D., Shier, A., \& Cafazzo, J. A. (2015). Technological aspects of hospital communication challenges: an observational study. Int J Qual Health Care, 27(3), 183-8. https://doi.org/10.1093/intqhc/mzv016

Raeisi, A., Rarani, M. A. \& Soltani F. (2019). Challenges of patient handover process in healthcare services: A systematic review. J Educ Health Promot, 8(173). 10.4103/jehp.jehp_460_18

Ramsay, N., Maresca, G., Tully, V. \& Campbell, K. (2018). Does a multidisciplinary approach have a beneficial effect on the development of a structured patient handover process between acute surgical wards in one of Scotland's largest teaching hospitals? BMJ Open Qual, 7(3), e000154. http://dx.doi.org/10.1136/bmjoq-2017-000154

Randmaa, M., Engström, M., Swenne, C. L. \& Mårtensson, G. (2017). The postoperative handover: a focus group interview study with nurse anaesthetists, anaesthesiologists and PACU nurses. BMJ Open, 7(8), e015038. http://dx.doi.org/10.1136/bmjopen-2016-015038

Rikos, N., Linardakis, M., Economou, C., Rovithis, M. \& Philalithis, A. (2019). The nurses' own views about the inter-shift handover process. Contemp Nurse, 55(1), 83-94. https://doi.org/10.1080/10376178.2019.1606723

Rikos, N., Linardakis, M., Rovithis, M. \& Philalithis, A. (2018). Features of recording practices and communication during nursing handover: a cluster analysis. Rev. esc. Enferm, 52, e03401. https://doi.org/10.1590/s1980-220x2018006203401

Rosenberg, A., Campbell, B. M., Feder, S., Minges, K., Hodshon, B., Chaudhry, S. I., \& Emerson, B. L. (2018). A taxonomy and cultural analysis of intrahospital patient transfers. Res Nurs Health. https://doi.org/10.1002/nur.21875

Sanchez, L. D., Chiu, D. T., Nathanson, L., Horng, S., Wolfe, R. E., Zeidel, M. L., \& Yang, J. J. (2017). A Model for Electronic Handoff Between the Emergency Department and Inpatient Units. J Emerg Med, 53(1), 142-150. https://doi.org/10.1016/j.jemermed.2017.03.027

Segall, N., Bonifacio, A. S., Barbeito, A., Schroeder, R. A., Perfect, S. R., Wright, M. C., \& Mark, J. B. (2016). Operating Room-to-ICU Patient Handovers: A Multidisciplinary Human-Centered Design Approach. Jt Comm J Qual Patient Saf, 42(9), 400-14. https://doi.org/10.1016/S1553-7250(16)42081-7

Stelfox, H. T., Leigh, J. P., Dodek, P. M., Turgeon, A. F., Forster, A. J., Lamontagne, F., \& Bagshaw, S. M. (2017). A multicenter prospective cohort study of patient transfers from the intensive care unit to the hospital ward. Intensive Care Med, 43(10), 1485-1494. https://doi.org/10.1007/s00134-017-4910-1

Tacchini-Jacquier, N., Waele, E., Urben, P., Turini, P. \& Verloo, H. (2020). Developing an EvidenceBased Nursing Handover Standard for a Multi-Site Public Hospital in Switzerland: Protocol for a Web-Based, Modified Delphi Study. JMIR Res. Proto, 9(1), e15910. $10.2196 / 15910$

Talley, D. A., Dunlap, E., Silverman, D., Katzer, S., Huffines, M., Dove, C., \& Tisherman, S. A. (2019). Improving Postoperative Handoff in a Surgical Intensive Care Unit. Crit Care Nurse, 39(5), e13-e21. https://doi.org/10.4037/ccn2019523

Thomson, H., Tourangeau, A., Jeffs, L. \& Puts, M. (2018). Factors affecting quality of nurse shift handover in the emergency department. $J$ Adv Nurs, 74(4), 876-886. https://doi.org/10.1111/jan.13499

Tobiano, G., Ting, C., Ryan, C., Jenkinson, K., Scott, L. \& Marshall, A. P. (2020). Front-line nurses' perceptions of intra-hospital handover. J Clin Nurs, 29(13-14), 2231-2238. https://doi.org/10.1111/jocn.15214

VanGraafeiland, B., Foronda, C., Vanderwagen, S., Allan, L., Bernier, M., Fishe, J., \& Jeffers J. M. (2018). Improving the handover and transport of critically ill pediatric patients. J Clin Nurs, 28(1-2), 56-65. https://doi.org/10.1111/jocn.14627

Vretare, L. L. \& Anderzén-Carlsson, A. (2020). The critical care nurse's perception of handover: A phenomenographic study. Intensive Crit Care Nurs, 58, 102807. https://doi.org/10.1016/j.iccn.2020.102807

World Heatlh Organization \& Joint Commission International. (2007). WHO Collaborating Centre for Patient Safety Solutions International: Communication During Patient Hand-Overs. Patient Safety Solution, 1(solution 3). https://www.who.int/patientsafety/solutions/patientsafety/PS-Solution3.pdf?ua=1

Yang, J. G. \& Zhang, J. (2016). Improving the postoperative handover process in the intensive care unit of a tertiary teaching hospital. J Clin Nurs, 25(7-8), 1062-72. https://doi.org/10.1111/jocn. 13115 
Research, Society and Development, v. 10, n. 9, e38910918153, 2021

(CC BY 4.0) | ISSN 2525-3409 | DOI: http://dx.doi.org/10.33448/rsd-v10i9.18153

Yegane, S. A. F., Shahrami, A., Hatamabadi, H. R. \& Hosseini-Zijoud, S. M. (2017). Clinical Information Transfer between EMS Staff and Emergency Medicine Assistants during Handover of Trauma Patients. Prehosp Disaster Med, 32(5), 541-547. https://doi.org/10.1017/S1049023X17006562

Yu, Z., Zhang, Y., Gu, Y., Xu, X. \& McArthur, A. (2017). Pediatric clinical handover: a best practice implementation project. JBI Database System Rev Implement Rep, 15(10), 2585-2596. https://doi.org/10.11124/JBISRIR-2016-003296

Zakrison, T. L., Rosenbloom, B., McFarlan, A., Jovicic, A., Soklaridis, S., Allen, C., \& Rizoli, S. (2016). Lost information during the handover of critically injured trauma patients: a mixed-methods study. BMJ Qual Saf, 25(12), 929-936. http://dx.doi.org/10.1136/bmjqs-2014-003903 